ESAIM: PROCEEDINGS, September 2005, Vol.14, 89-99

Eric Cancès \& Jean-Frédéric Gerbeau, Editors

DOI: $10.1051 /$ proc:2005008

\title{
DEFECTIVE BOUNDARY CONDITIONS APPLIED TO MULTISCALE ANALYSIS OF BLOOD FLOW*
}

\author{
Fernández M. ${ }^{1}$, Moura A. ${ }^{2}$ And Vergara C. ${ }^{2}$
}

\begin{abstract}
In hemodynamics, the prescription of suitable boundary conditions for the Navier-Stokes equations (3D model) on the artificial sections (i.e. the parts of the boundary not corresponding to the physical artery wall) is critical. A first solution is to prescribe experimental data, whenever available from specific measurements, or we can use reduced models, i.e. one-dimensional (1D) or zero-dimensional (0D) models, to get the proper interface conditions accounting for global behavior $($ see $[4,5])$. In this work we couple a $0 \mathrm{D}$ model with a $3 \mathrm{D}$ local model of a non-compliant cylindric vessel. We propose two techniques. In the first approach the reduced model provides the mean pressure to be imposed as defective boundary condition to the 3D model, which conversely will make ready the flow rate to the reduced model. In the second strategy the type of data to be exchanged is reversed.

Mean value conditions are not natural to Navier-Stokes equations, which would need a vector condition at each point of $\Gamma_{j}$. Special techniques have to be implemented. For what concerns the mean pressure problem, we follow the approach proposed in [9], that suggests to impose on the artificial section some natural (Neumann) conditions obtained from a suitable variational formulation. For the flow rate problem, we use the augmented formulation proposed in [2] and [16]. In these works, the flux conditions are regarded as constraints to be fulfilled by the solution by introducing a Lagrange multiplier for each defective condition.
\end{abstract}

\section{INTRODUCTION}

Over the last years, the advances on fluid-dynamics numerical simulations, together with the development of new technologies in experimental data acquisition have allowed to obtain quantitative information of the blood flow behavior. For instance, measures of shear stresses in the vessel wall, identification of recirculation zones in vascular districts, the blood-vessel mechanical interaction or the adaptation of the vascular walls after surgical intervention (see for example [12]). Specifically, research focused on the determination of the velocity and pressure field, based on the knowledge of some global quantities as the flux or the mean pressure on specific boundaries of the vascular district under study.

In medium and large vessels, blood behaves as an incompressible Newtonian fluid. The incompressible Navier-Stokes equations are then a mathematical model capable to provide the velocity and the pressure of the blood. Nowadays, three-dimensional simulations of blood flow based on these equations are a common practice [11]. Obviously, these simulations require the enforcement of specific data on the artificial boundary

\footnotetext{
* This work has been supported by the European Network HAEMODEL - Mathematical Modelling of the Cardiovascular System under contract HPRN - CT - 2002-002670.

1 Institut National de Recherche en Informatique et en Automatique; e-mail: miguel.fernandez@inria.fr

2 MOX (Modeling and Scientific Computing) Department of Mathematics, Politecnico di Milano;

e-mail: alexandra.moura@mate.polimi.it \& christian.vergara@mate.polimi.it
} 
sections (i.e. the part of the boundary not corresponding to the wall). Typically one imposes standard Dirichlet or Neumann boundary conditions. However, the human cardiovascular system is formed by a closed network of vessels with a high level of interdependency. Thus, a realistic numerical simulation of the blood flow in a local district may not be fully accomplished if the interactions with the systemic haemodynamics are neglected. The prescription of suitable boundary conditions on the artificial sections is critical and standard conditions are often inappropriate. A first solution is to resort to experimental measurements. To be suitable for a Navier-Stokes problem, however, the data should provide information on the pointwise variation of fluid quantities (such as velocity or stresses). This is seldom possible. When data measurements are not available, we can use reduced models of the cardiovascular system and prescribe the boundary conditions for the artificial sections of the 3D model by coupling these different models. This approach leads to the geometrical multiscale modeling of the circulatory system. The main principle of such a modelization (see, for instance, $[8,10,13,15]$ ) consists in approximating the different parts of the cardiovascular system with different levels of accuracy, which leads to different levels of computational complexity. The simpler models are derived from the 3D model (described by the Navier-Stokes equations) by making some simplifications and assumptions (see [4]). The one-dimensional (1D) model consists in an hyperbolic system and provides useful information on the wave propagation phenomena in the arterial tree. The zero-dimensional (0D) or lumped parameters model, obtained by making some further assumptions, reduces to a system of ordinary differential equations. It allows to describe global characteristics of the human circulatory system, as the heart action or the presence of the venous and capillary bed. By coupling these different models one can obtain both global and local information on the blood flow behavior, in particular, it is possible to observe the influence of the systemic circulation on the particular district of interest and vice-versa. There are several kind of coupled models proposed in literature, depending on the role given to each part of the model. For instance a 1D model can act as an absorbing boundary for a 3D fluid-structure model (see [5]), and a 0D model can replace the systemic circulation coupled with a $3 \mathrm{D}$ model $($ see $[8,13])$. In $[7,8]$ we find some examples of coupling between reduced models.

In the present work we couple a lumped parameters model $(0 \mathrm{D})$ of the circulatory system with a $3 \mathrm{D}$ model of a rigid cylindrical vessel (described by the Navier-Stokes equations). The coupling is performed enforcing the continuity of the flow rate and of the mean pressure on the artificial interface sections (denoted by $\Gamma_{j}$ ).

We here propose two different coupling strategies, to be used in the frame of an iterative procedure. In the first approach the network provides a mean pressure value as defective boundary condition on $\Gamma_{j}$, while the $3 \mathrm{D}$ model returns the flow rate through $\Gamma_{j}$ to the network. Then we consider the reverse situation. That is, the latest pressure computed by the Navier-Stokes solver is passed to the reduced model which updates the mass flux through $\Gamma_{j}$. In both cases the Navier-Stokes equations are equipped with non standard conditions, which have to be properly set in order to have a well posed problem. For what concerns the mean pressure problem, in this paper we follow the approach proposed in [9], that suggests to impose on the artificial section some natural (Neumann) conditions obtained from a suitable variational formulation (see Section 2). For the flow rate problem, we use the augmented formulation proposed in [2] and [16]. In these papers, the flux conditions are regarded as constraints to be fulfilled by the solution and are implemented by introducing a Lagrange multiplier for each defective condition.

In Section 1 we present two algorithms for the resolution of the flux problem: the GMRes+Schur Complement algorithm proposed in [16] and its inexact version. Section 2 provides a mathematical framework for coupling a lumped parameters model with a 3D Navier-Stokes problem, including a discussion of some coupling strategies. Moreover, in Section 3 we present some numerical results with application to multiscale modeling of the blood flow. 


\section{Flux BOUndary CONDITIONS}

\subsection{The Model}

Let us consider the computational domain $\Omega$ in Figure 1, in which we solve the Navier-Stokes equations

$$
\left\{\begin{array}{rlrl}
\rho\left(\frac{\partial \boldsymbol{u}}{\partial t}+\boldsymbol{u} \cdot \nabla \boldsymbol{u}\right)-\nu \Delta \boldsymbol{u}+\nabla p & =\boldsymbol{f}, & & \text { in } \Omega \times(0, T), \\
\operatorname{div} \boldsymbol{u} & =0, & & \text { in } \Omega \times(0, T), \\
\boldsymbol{u}(\cdot, 0) & =\boldsymbol{u}_{0}, & \text { in } \Omega .
\end{array}\right.
$$

Here, $\rho$ stands for the fluid density, $\boldsymbol{u}$ for the velocity field, $p$ for the pressure, $\nu$ for the dynamic viscosity and $\boldsymbol{f}, \boldsymbol{u}_{0}$ denote a given source term and initial velocity. Problem (1) has to be completed with suitable boundary conditions. In particular we set a no-slip condition on the vessel wall $\Sigma$ (the wall is assumed to be rigid),

$$
\left.\boldsymbol{u}\right|_{\Sigma}=\mathbf{0} \quad \forall t \in(0, T)
$$

The boundary sections $\Gamma_{j}$ correspond to artificial boundaries, on which we want to impose:

$$
\int_{\Gamma_{j}} \boldsymbol{u} \cdot \boldsymbol{n} d \sigma=F_{j} \quad j=1, \ldots, n .
$$

Let us notice that, due to mass conservation (and since the domain is fixed), we cannot impose the flux on all the artificial boundaries, but on all but one. For example, on $\Gamma_{0}$ we impose an homogeneous Neumann condition (see [2]). Since the Navier Stokes problem requires pointwise boundary conditions on $\Gamma_{j}$, the prescription of the fluxes (3) is not sufficient to close problem (1). However, we can impose these average conditions through a set of $n$ Lagrange multipliers, seeing conditions (3) as constraints satisfied by the solution (see [2]). Let us set

$$
\boldsymbol{V}=\left\{\boldsymbol{v} \in \boldsymbol{H}^{1}(\Omega):\left.\boldsymbol{v}\right|_{\Sigma}=\mathbf{0}\right\}
$$

We obtain the following variational formulation for the problem given by (1), (2) and $(3)$ (see $[2,16])$ :

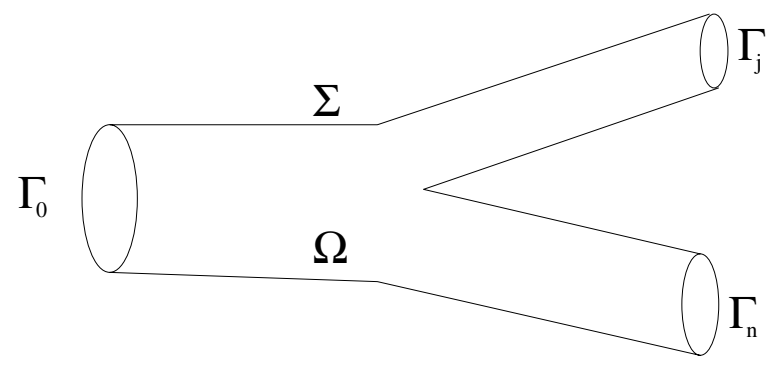

Figure 1: Generic vascular district $\boldsymbol{\Omega}$.

Problem 1 Given $\boldsymbol{u}_{0} \in \boldsymbol{V}, \boldsymbol{f} \in L^{2}\left(0, T ; \boldsymbol{L}^{2}(\Omega)\right)$ and $\boldsymbol{F} \in\left[L^{2}(0, T)\right]^{n}$, such that $\int_{\Gamma_{j}} \boldsymbol{u}_{0} \cdot \boldsymbol{n} d \sigma=F_{j}(0)$, find $\boldsymbol{u} \in L^{2}(0, T ; \boldsymbol{V}), p \in L^{2}\left(0, T ; L^{2}(\Omega)\right)$ and $\boldsymbol{\lambda} \in\left[L^{2}(0, T)\right]^{n}$ such that for all $t \in(0, T)$

$$
\left\{\begin{aligned}
\rho\left(\frac{\partial \boldsymbol{u}}{\partial t}+(\boldsymbol{u} \cdot \nabla) \boldsymbol{u}, \boldsymbol{v}\right)+\nu(\nabla \boldsymbol{u}, \nabla \boldsymbol{v})-(\operatorname{div} \boldsymbol{v}, p)+\sum_{j=1}^{n} \lambda_{j} \int_{\Gamma_{j}} \boldsymbol{v} \cdot \boldsymbol{n} d \sigma & =(\boldsymbol{f}, \boldsymbol{v}) \\
(\operatorname{div} \boldsymbol{u}, q) & =0 \\
\boldsymbol{u}(0) & =\boldsymbol{u}_{0}, \quad \forall j=1, \ldots, n, \\
\int_{\Gamma_{j}} \boldsymbol{u} \cdot \boldsymbol{n} d \sigma & =F_{j}, \quad \forall j,
\end{aligned}\right.
$$


for all $\boldsymbol{v} \in \boldsymbol{V}$ and $q \in L^{2}(\Omega)$.

The Lagrange multipliers have the physical meaning of normal stresses, constant on each $\Gamma_{j}$ (see [2]). Under suitable hypothesis on the data, Problem 1 is locally (in time) well posed (see [16]).

\subsection{Numerical Treatment}

At the numerical level the problem can be solved by augmenting the algebraic system derived from a finite element discretization with the equations given by (3), and with the new unknowns $\lambda_{j}, j=1, \ldots, n$. More precisely, we introduce an approximation based on finite elements, choosing appropriate approximation spaces $\boldsymbol{V}_{h} \subset \boldsymbol{V}$ e $Q_{h} \subset L^{2}(\Omega)$, of finite dimension $N$ and $M$, respectively, and satisfying the LBB or inf-sup condition. We look for approximate solutions $\boldsymbol{u}_{h}$ and $p_{h}$ such that

$$
\boldsymbol{u}_{h}(\boldsymbol{x}, t)=\sum_{i=1}^{N} u_{j}(t) \boldsymbol{\psi}_{j}(\boldsymbol{x}), \quad p_{h}(\boldsymbol{x}, t)=\sum_{k=1}^{M} p_{k}(t) \zeta_{k}(\boldsymbol{x}),
$$

where $\boldsymbol{\psi}_{j}$ and $\zeta_{k}$ denote, respectively, the basis functions of $\boldsymbol{V}_{h}$ and $Q_{h}$. The system related to the spatial discretization of Problem 1 is then

$$
\left\{\begin{aligned}
M \frac{d \boldsymbol{U}(t)}{d t}+A \boldsymbol{U}(t)+B(\boldsymbol{U}(t)) \boldsymbol{U}(t)+C^{t} \boldsymbol{P}(t)+\Phi^{t} \boldsymbol{\Lambda}(t) & =\boldsymbol{H}(t), \\
C \boldsymbol{U}(t) & =\mathbf{0} \\
\Phi \boldsymbol{U}(t) & =\boldsymbol{F}(t),
\end{aligned}\right.
$$

where $M_{i j}=\left(\boldsymbol{\psi}_{i}, \boldsymbol{\psi}_{j}\right), A_{i j}=a\left(\boldsymbol{\psi}_{i}, \boldsymbol{\psi}_{j}\right)$ and $B_{i j}=b\left(\boldsymbol{\psi}_{i}, \boldsymbol{\psi}_{j}, \boldsymbol{\psi}_{k}\right)$, for $i, j, k=1, \ldots, N ; \quad C_{k l}=c\left(\zeta_{k}, \boldsymbol{\psi}_{l}\right)$, for $k=1, \ldots, M$ and $l=1, \ldots, N ; \quad \boldsymbol{U}(t)=\left\{u_{j}(t)\right\}_{j=1, \ldots, 3 N}$, and $\boldsymbol{P}(t)=\left\{p_{k}(t)\right\}_{j=1, \ldots, M}$, are the vectors of the unknown coefficients; $\Phi_{r j}=\int_{\Gamma_{r}} \boldsymbol{\psi}_{j} \cdot \boldsymbol{n} d \sigma$, for $r=1, \ldots, n$ and $j=1, \ldots, N, \Lambda(t)=\left\{\lambda_{r h}(t)\right\}_{r=1, \ldots, n}$, the vector of the approximate Lagrange multipliers. Finally, we set $\boldsymbol{H}(t)=\left\{H_{j}(t)=\boldsymbol{f}(t), \boldsymbol{\psi}_{j}\right\}_{j=1, \ldots, N}$ and $\boldsymbol{F}(t)=\left\{\left(F_{i}(t)\right)\right\}_{i=1, \ldots, n}$. Treating in a semi-implicit way the non linear convective term and applying a backward Euler formula for the time derivative, we obtain the following fully algebraic problem

$$
\left(\begin{array}{ccc}
K & C^{t} & \Phi^{t} \\
C & 0 & 0 \\
\Phi & 0 & 0
\end{array}\right)\left(\begin{array}{c}
\boldsymbol{U}^{n+1} \\
\boldsymbol{P}^{n+1} \\
\boldsymbol{\Lambda}^{n+1}
\end{array}\right)=\left(\begin{array}{c}
\widetilde{\boldsymbol{H}}^{n+1} \\
\mathbf{0} \\
\boldsymbol{F}^{n+1}
\end{array}\right)
$$

where $K\left(\boldsymbol{U}^{n}\right)=\frac{1}{\Delta t} M+A+B\left(\boldsymbol{U}^{n}\right)$ and $\widetilde{\boldsymbol{H}}^{n+1}=\boldsymbol{H}^{n+1}+\frac{1}{\Delta t} \boldsymbol{U}^{n}$. In analogy with [2], let us write (5) in an alternative way

with

$$
\left(\begin{array}{cc}
S & \widetilde{\Phi}^{t} \\
\widetilde{\Phi} & 0
\end{array}\right)=\left(\begin{array}{c}
\boldsymbol{X}^{n+1} \\
\boldsymbol{\Lambda}^{n+1}
\end{array}\right)=\left(\begin{array}{c}
\widetilde{\boldsymbol{H}}^{n+1} \\
\boldsymbol{F}^{n+1}
\end{array}\right)
$$

$$
\begin{array}{r}
S=\left(\begin{array}{cc}
K & C^{t} \\
C & 0
\end{array}\right) \in \mathbb{R}^{(3 N+M) \times(3 N+M),} \quad \widetilde{\Phi}=\left(\begin{array}{cc}
\Phi & 0
\end{array}\right) \in \mathbb{R}^{n \times(3 N+M),} \\
\boldsymbol{X}=\left(\begin{array}{c}
\boldsymbol{U} \\
\boldsymbol{P}
\end{array}\right) \in \mathbb{R}^{3 N+M}, \quad \widetilde{\widetilde{\boldsymbol{H}}}^{n+1}=\left(\begin{array}{c}
\widetilde{\boldsymbol{H}}^{n+1} \\
\mathbf{0}
\end{array}\right) \in \mathbb{R}^{3 N+M} .
\end{array}
$$

Since LBB condition holds, $S$ is non singular and we can eliminate the unknown $\boldsymbol{X}$ from (6), yielding

$$
\widetilde{\Phi} S^{-1} \widetilde{\Phi}^{t} \boldsymbol{\Lambda}=\widetilde{\Phi} S^{-1} \widetilde{\boldsymbol{H}}-\boldsymbol{F} .
$$

Let us notice that each application of the inverse operator $S^{-1}$ is nothing but the resolution of a classical Navier-Stokes problem, which can be performed, for instance, using an inexact factorization method (see [17]) 
or with block factorized preconditioners (see [18]). In particular, in this work we used the fast preconditioner proposed and analyzed in [3]. The matrix $R=\widetilde{\Phi} S^{-1} \widetilde{\Phi}^{t}$ is positive semidefinite (see [2]). So, (8) can be solved using GMRes iterations, as described in the following Algorithm.

$$
\begin{aligned}
& \boldsymbol{\Lambda}_{0}=\left(\lambda_{01}, \ldots, \lambda_{0 n}\right) \text { is given } \\
& S \boldsymbol{X}_{0}=\widetilde{\widetilde{H}}-\widetilde{\Phi}^{t} \boldsymbol{\Lambda}_{0}(*) \\
& \boldsymbol{r}_{0}=\widetilde{\Phi} \boldsymbol{X}_{0}-\boldsymbol{F} \\
& \boldsymbol{v}_{1}=\frac{\boldsymbol{r}_{0}}{\left\|\boldsymbol{r}_{0}\right\|} \\
& \text { for } j=1, \ldots, n \\
& \boldsymbol{\eta}_{j}=\widetilde{\Phi}^{t} \boldsymbol{v}_{j} \\
& S \boldsymbol{\xi}_{j}=\boldsymbol{\eta}_{j}(* *) \\
& \boldsymbol{w}_{j}=\widetilde{\Phi} \boldsymbol{\xi}_{j} \\
& \text { for } i=1, \ldots, j \\
& h_{i j}=\left(\boldsymbol{w}_{j}, \boldsymbol{v}_{i}\right) \\
& \boldsymbol{w}_{j}=\boldsymbol{w}_{j}-h_{i j} \boldsymbol{v}_{i} \\
& \text { end } \\
& h_{j+1, j}=\left\|\boldsymbol{w}_{j}\right\| \\
& \text { if } h_{j+1, j}=0 \\
& n=j \text { go to }(\dagger) \\
& \text { else } \boldsymbol{v}_{j+1}=\frac{\boldsymbol{w}_{j}}{h_{j+1, j}} \\
& \text { end } \\
& \text { end } \\
& (\dagger) \boldsymbol{y}=\min \|\| \boldsymbol{r}_{0}\left\|\boldsymbol{e}_{1}-H_{n} \boldsymbol{y}\right\| \\
& \boldsymbol{\Lambda}=\boldsymbol{\Lambda}_{0}+V \boldsymbol{y} \\
& S \boldsymbol{X}=\widetilde{\widetilde{\boldsymbol{H}}}-\widetilde{\Phi^{t}} \boldsymbol{\Lambda}(* * *)
\end{aligned}
$$

Here, $V$ is the matrix whose columns are the vectors $\boldsymbol{v}_{1}, \ldots, \boldsymbol{v}_{n}$ and $H_{n}$ the $(n+1) \times n$ matrix whose entries are $h_{i j}$.

Based on the previous algorithm, an existing Navier-Stokes solver may be readily extended to treat a problem involving flux boundary conditions. The computational cost of this procedure depends mainly on the number of Navier-Stokes problems we have to solve: one to obtain the initial residual (indicated with $\left(^{*}\right.$ ) in the algorithm), $n$ (i.e. the dimension of the unknown $\boldsymbol{\Lambda}$ ) to obtain $\boldsymbol{\xi}_{j}(* *)$, since we notice that GMRes algorithm converges exactly in $n$ iterations (as in the exact arithmetic case), and one to obtain the final solution $\boldsymbol{X}(* * *)$. Nevertheless, the last Navier-Stokes computation is trivial, since from $(*)$ we have $\widetilde{\widetilde{H}}=S \boldsymbol{X}_{0}+\widetilde{\Phi^{t}} \boldsymbol{\Lambda}_{0}$ and substituting in (***) we obtain:

$$
\boldsymbol{X}=\boldsymbol{X}_{0}-S^{-1} \Phi^{t}\left(\boldsymbol{\Lambda}-\boldsymbol{\Lambda}_{0}\right)=\boldsymbol{X}_{0}-S^{-1} \Phi^{t} V \boldsymbol{y}=\boldsymbol{X}_{0}-\Xi \boldsymbol{y}
$$

where $\Xi$ is the matrix whose columns are the vectors $\boldsymbol{\xi}_{1}, \ldots, \boldsymbol{\xi}_{n}$. Definitively, we have to solve $n+1$ Navier-Stokes problems at each time step.

We notice that whenever matrix $S$ does not depend on time (i.e. for the Stokes problem) the solution of $(* *)$ can be computed outside of the temporal loop. In fact, if we know the solution of the $n$ problems $S \boldsymbol{\theta}_{i}=\widetilde{\Phi}^{t} \boldsymbol{\beta}_{i} j=i, \ldots, n$, being $\boldsymbol{\beta}_{1}, \ldots, \boldsymbol{\beta}_{n}$ a basis of $\mathbb{R}^{n}$, we can construct the unknown $\boldsymbol{\xi}_{j}$ since:

$$
\boldsymbol{\xi}_{j}=\sum_{i=1}^{n} v_{j i} \boldsymbol{\theta}_{i} .
$$

In the Navier-Stokes case, the matrix $S$ is time dependent since $S=S\left(\boldsymbol{U}^{n}\right)$. However, the previous strategy can be extended using approximation of the matrix $S$, for example by setting $S=S\left(\boldsymbol{U}^{0}\right)$ at each time step. 
This leads to the inexact GMRes+Schur complement method. The computational cost of this strategy is equal to $n$ steady and 1 unsteady Navier Stokes problem (the first one in Algorithm 1). This reduced computational cost is obtained with price of accepting a less accurate solution. The numerical results reported in Section 3 show that the inexact method provides good approximations. Finally, an hybrid approach consists in updating the matrix $S$ every $m$ time steps, with $m \geq 1$. For $m=1$ we recover the GMRES+Schur complement algorithm, while for $m=\infty$ we recover the inexact $\bar{G} M R E S+$ Schur complement algorithm.

\section{Multiscale Modeling of Blood Flow}

We consider equations (1) and (2) as the 3D model describing the blood flow in the local region of interest. In order to prescribe data on the artificial boundaries we couple the 3D model with a lumped parameters description of the cardiovascular system. In this section we review the $0 \mathrm{D}$ model and the coupling strategies.

\subsection{The Lumped Parameters Model}

There are two kinds of reduced models that can be used to describe the human circulatory system at a low computational cost: the 1D models and the lumped parameters (0D) ones. These models describe different features of the human cardiovascular system.

Starting from the 3D incompressible Navier-Stokes equations in a compliant straight cylindrical vessel, under certain assumptions, one can derive a one-dimensional (1D) model, stated in terms of hyperbolic equations along the axial coordinate of the vessel. One-dimensional models have shown to provide useful information at low computational cost, in particular, these models are very well adapted for the simulation of wave propagations in the arterial tree [7].

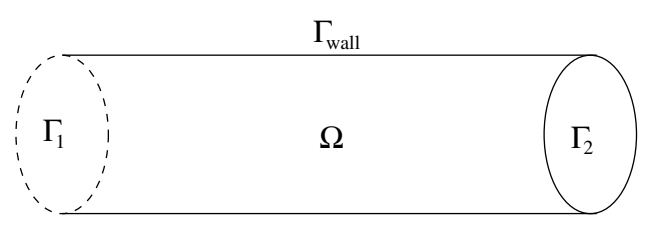

Figure 2: Compliant cylindrical vessel.

The lumped parameters models are expressed in terms of systems of ordinary differential equations (ODE), describing the averaged (in time) mass and flow rate in a specific terminal compartment of the circulatory system. Since they do not account for variations in space, they are often called zero-dimensional (0D) models. They can be derived starting from the 1D model by integrating the equations in space. Indeed, after linearization, we obtain [4]:

$$
\left\{\begin{aligned}
C \frac{d \hat{P}}{d t}+Q_{2}-Q_{1} & =0 \\
L \frac{d \hat{Q}}{d t}+R \hat{Q}+P_{2}-P_{1} & =0
\end{aligned}\right.
$$

where the state variables are the flow rate $\hat{Q}$ and the mean pressure $\hat{P}$ and $R, L$ and $C$ are given constants. The values $Q_{1}, P_{1}, Q_{2}$ and $P_{2}$ are obtained from the values of the velocity and pressure, respectively on $\Gamma_{1}$ and on $\Gamma_{2}$. Two of these values are derived from the boundary conditions of the 3D Navier-Stokes equations: from a Dirichlet condition on $\Gamma_{j}$ we obtain the value of $Q_{j}$, while a Neumann condition gives the value of $P_{j}$. The two remaining values are set equal to the state variables; this is a reasonable approximation assuming that the district is sufficiently small. Thus, according to the boundary conditions on the artificial sections of the 3D model, there are four different possibilities for the lumped description of the cylinder. For example, if we prescribe a Neumann condition on $\Gamma_{1}$ and a Dirichlet condition on $\Gamma_{2}$, the resulting lumped parameters model is shown in Figure 3. Let us notice that where the flux is prescribed there is a capacitance, since the state variable is the pressure, while where the pressure is imposed there is an inductance. 


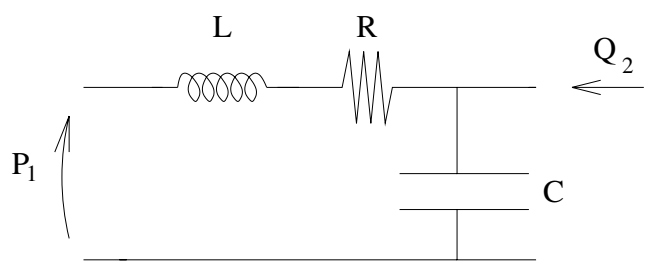

Figure 3: Example of a network representing a compliant cylindrical vessel.

By jointing together the lumped description of the various cylindric vessels composing a more complex system, we obtain a 0D model for a more complex geometry. In [4,15] other lumped parameters model are given, describing other compartments of the circulatory system, such as the heart, the venous bed or the pulmonary tree. For instance in the Windkessel model, the heart compartments are described with a pressure source, capacitances changing in time and diodes modeling the valves.

In general, a lumped parameters model describing the whole circulatory system can be described by an ODE's system as

$$
\left\{\begin{array}{cll}
\frac{d \mathbf{y}}{d t}=\mathbf{A y}+\mathbf{b}+\mathbf{r} & , t>0 \\
\mathbf{y}=\mathbf{y}_{\mathbf{0}} & , t=0
\end{array}\right.
$$

where $\mathbf{y}$ is the vector of the state variables, $\mathbf{A}$ is the system matrix, $\mathbf{b}$ derives from the $3 \mathrm{D}$ model boundary conditions, $\mathbf{r}(t)$ accounts for the pressure source term and $\mathbf{y}_{0}$ is the given initiate data.

The lumped parameters model can be regarded as an electric network. Indeed the flow rate can be seen as the electric current and the mean pressure as the voltage. Furthermore, the resistance $R$ is related with the blood viscosity, the inductance $L$ with the blood inertia and the capacitance $C$ with the wall compliance of the cylindric artery. In fact, constants $R, L$ and $C$ depend on the physical characteristics of the artery, such as length, radius, thickness of the wall, density, viscosity and Young Modulus.

\subsection{The coupling}

We assume that system (10) describes the entire (or a part) circulatory system and we substitute a part of that network with its correspondent $3 \mathrm{D}$ description. In an iterative frame to the solution of the coupled problem, the $3 \mathrm{D}$ model provides point-wise information on the velocity and pressure which could be integrated to obtain the average data to be prescribed to the 0D model. For each interface section $\Gamma_{j}$ there are two choices:

- The $3 \mathrm{D}$ model provides the flow rate $Q_{j}$ to the network while the latter is used to compute the mean pressure $P_{j}$ to be input to $3 \mathrm{D}$ model. In this case we have a mean pressure boundary condition for the Navier-Stokes equations in the interface boundary. To this aim we have followed the do nothing approach proposed in [9]. Therein, we pose $-p \mathbf{n}+\left.\nu(\nabla \mathbf{u}) \mathbf{n}\right|_{\Gamma_{j}}=P_{j} \mathbf{n}$ as boundary condition for the Navier-Stokes problem on $\Gamma_{j}$. In this case a capacitance has to be gathered on the interface with $\Gamma_{j}$.

- The 3D model provides the mean pressure $P_{j}$ to the network while the latter gives the flow rate $Q_{j}$ to the 3D model. In this case we have a flux problem for the Navier Stokes equations and we follow the strategy of Section 1. In this case an inductance must be allocated on the 0D model at the interface $\Gamma_{j}$.

According to the particular district described by the 3D model, namely the number of interface boundaries, all combinations of these two coupling strategies are possible for each boundary section. The only observation is that if the $3 \mathrm{D}$ model does not account for wall compliance then the flux can be imposed on all boundary sections but one.

In [14] the well posedness of the problem of coupling a 0D model with the 3D Navier-Stokes model in a rigid domain using the first coupling strategy has been proved. It was checked that the same coupling problem is also well posed for the second coupling strategy, by using the same fixed-point strategy in [14] and the fact that the flux problem for the Navier-Stokes equations is itself well posed (see [16]). 


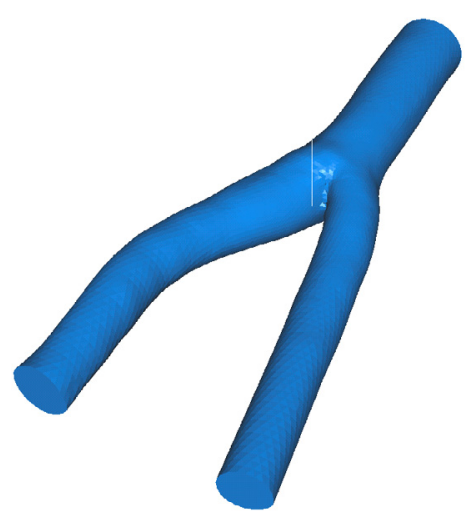

Figure 4: Carotid district.

\subsection{Numerical approximation}

In this paragraph we summarize the numerical approximation of the coupling (see [13]). The numerical treatment is based on a splitting strategy: at each time step each model provides the necessary data to the other one. The usual Galerkin finite-element method is applied to solve the Navier-Stokes equations. The explicit forward Euler method is applied to solve the ODE system corresponding to the network.

Let $\mathbf{y}^{n}$ and $\mathbf{x}^{n}=(\mathbf{u}, p)^{n}$ (or $\mathbf{x}^{n}=(\mathbf{u}, p, \lambda)^{n}$ in the case of the flux problem) be the solutions at time step $t^{n}$ of the lumped parameters ODE's system (10) and of the Navier-Stokes equation, respectively. The algebraic system arising from the discretization of the coupled problem reads (see [13])

$$
\left[\begin{array}{cc}
\mathcal{N}_{1} & \mathcal{N}_{\mathcal{B C}_{1}} \\
\mathcal{N S}_{\mathcal{B C}} & \mathcal{N S}_{1}
\end{array}\right]\left[\begin{array}{c}
\mathbf{y}^{n+1} \\
\mathbf{x}^{n+1}
\end{array}\right]=\left[\begin{array}{cc}
\mathcal{N}_{2} & \mathcal{N}_{\mathcal{B C}_{2}} \\
\mathcal{N S}_{\mathcal{B C}} & \mathcal{N S}_{2}
\end{array}\right]\left[\begin{array}{l}
\mathbf{y}^{n} \\
\mathbf{x}^{n}
\end{array}\right]+\left[\begin{array}{l}
\mathbf{g} \\
\mathbf{0}
\end{array}\right]
$$

where $\mathcal{N}_{1}$ is related to the discretization of term $\frac{d \mathbf{y}}{d t}, \mathcal{N}_{2}$ is related to the discretization of matrix $\mathbf{A}$, $\mathbf{g}$ corresponds to the approximation of $\mathbf{r}, \mathcal{N}_{\mathcal{B C}_{1}}$ and $\mathcal{N}_{\mathcal{B C}_{2}}$ are due to the discretization of term $\mathbf{b}, \mathcal{N S}_{\mathcal{B C}}$ is related to the discretization of the boundary conditions for the Navier-Stokes equations, $\mathcal{N S}_{1}$ and $\mathcal{N S}_{2}$ correspond to the approximation of the Navier-Stokes problem.

The coupled system (11) is solved by a Gauss-Seidel iterative method. Since the time discretization of the lumped parameters model is explicit, it turns out that $\mathcal{N}_{\mathcal{B C}}{ }_{1}=0$ and thus the left-hand side matrix of system (11) is lower triangular. So the Gauss-Seidel method converges in one iteration. In other words, the computation of the two discretized models is completely separated at each time step.

\section{Numerical Results}

The first simulation concerns the carotid geometry in Figure 4. We impose a sinusoidal flux with period $T=1 \mathrm{sec}$ on both distal branches (with amplitude $A=0.01 \mathrm{~cm} / \mathrm{sec}$ on the left one and $A=0.005 \mathrm{~cm} / \mathrm{sec}$ on the other one). We use the GMRes+Schur complement algorithm. The velocity at two different time steps on a longitudinal section is shown in Figure 5. Let us notice the flat velocity profile at the begin of the period (left) 

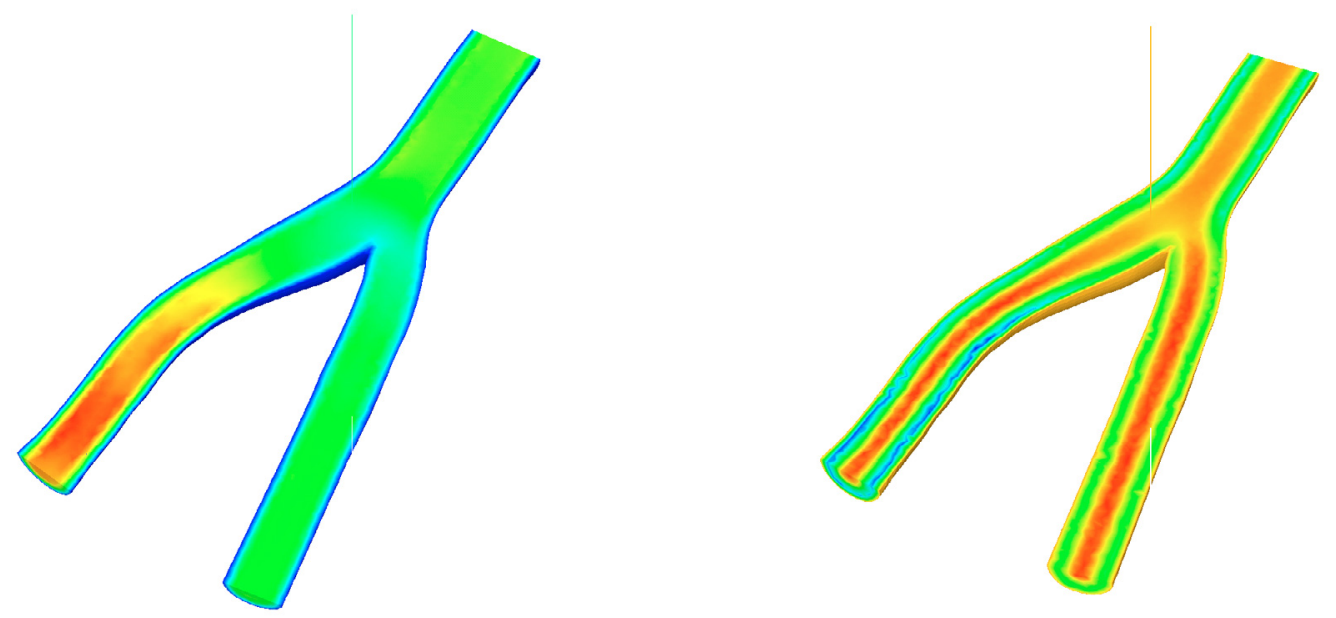

Figure 5: Velocity at $t=0.08$ (left) and $t=0.28$ (right).

and the zones of inversion of the fluid, as expected from the Womersley theory, at $t=0.28$ (right). Moreover, solving the same problem with the inexact version of the GMRes+Schur complement algorithm, we have the following relative error:

$$
E_{r}=\frac{\left\|u_{e x}(\boldsymbol{x}, t)-u_{\text {inex }}(\boldsymbol{x}, t)\right\|_{L^{\infty}\left(0, T ; L^{\infty}(\Omega)\right)}}{\left\|u_{e x}(\boldsymbol{x}, t)\right\|_{L^{\infty}\left(0, T ; L^{\infty}(\Omega)\right)}}=7.6 \cdot 10^{-4}
$$

By this error estimate the inexact version of Algorithm 1 seems to be very accurate. In Table 1 we can also see that with the inexact algorithm the computational cost heavily decreases.

\begin{tabular}{|r|c|}
\hline & CPU time $(\mathrm{sec})$ \\
\hline exact & 2842 \\
\hline inexact & 978 \\
\hline
\end{tabular}

TABle 1. CPU time for one time-step.

Next, we present some numerical results obtained coupling a non-compliant cylinder with the simple network of Figure 6, which has a pressure source of type $\mathbf{U}(t)=c+\cos (2 \pi t)$, representing a periodic action.

In Tables 2 and 3 we present the results obtained with both coupling strategies, comparing them with the results obtained by substituting the $3 \mathrm{D}$ model with its equivalent lumped parameters description. We observe that the equivalent 0D model of the cylinder is not the same for the two coupling strategies. We conclude that both strategies lead to good results, i.e. in both cases the flow behavior on the 3D model evidences the periodic impulse of the network accurately. 


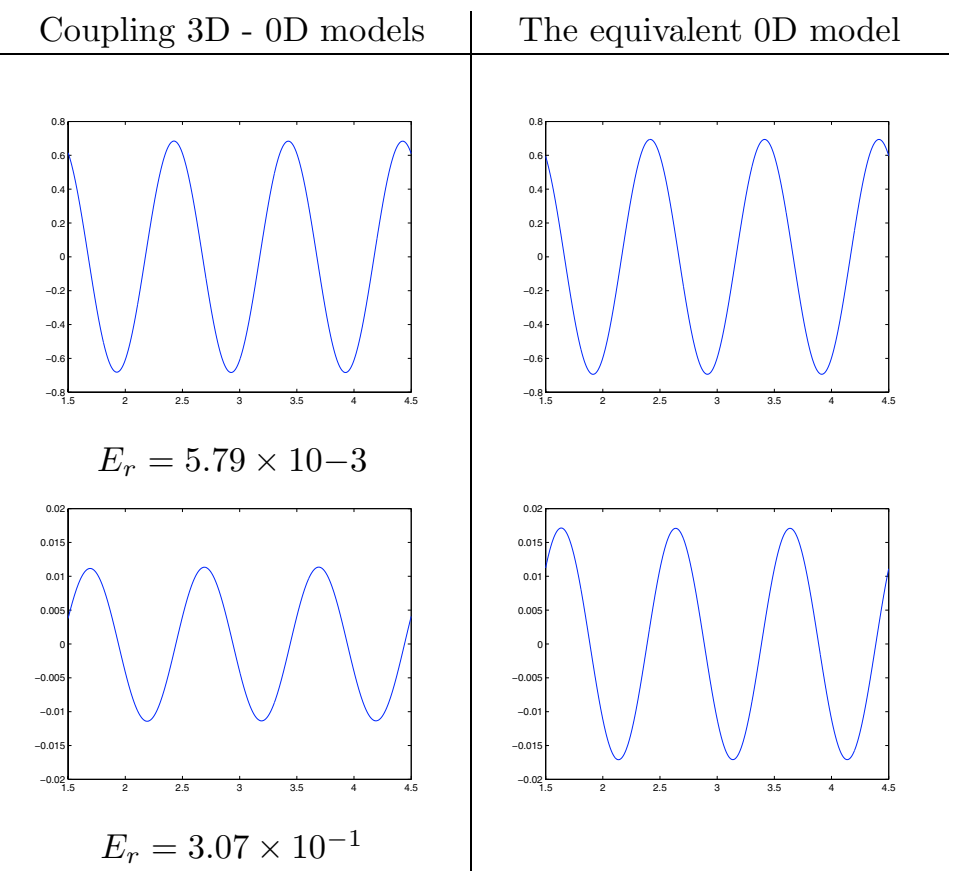

TABLE 2. Comparative results for the mean pressure drop (top) and the flow rate (bottom) using the strategy of imposing the mean pressure on the 3D model.

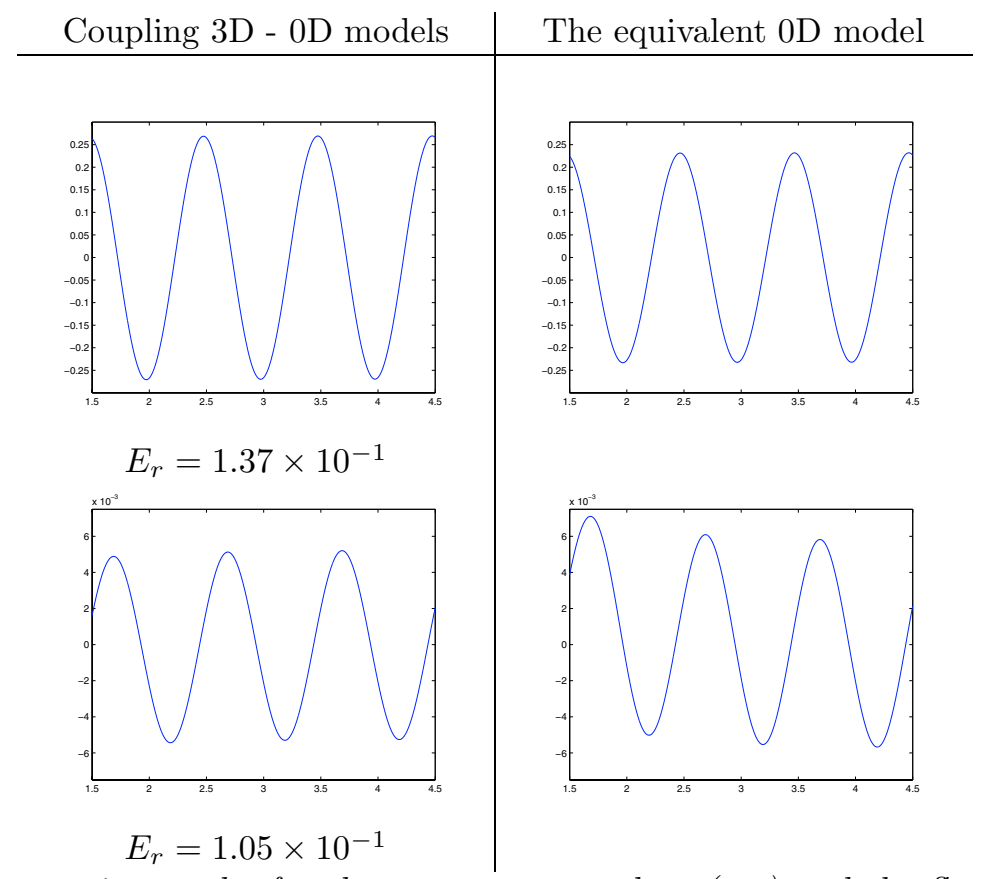

TABLE 3. Comparative results for the mean pressure drop (top) and the flow rate (bottom) using the strategy of imposing the flow rate on the 3D model. 


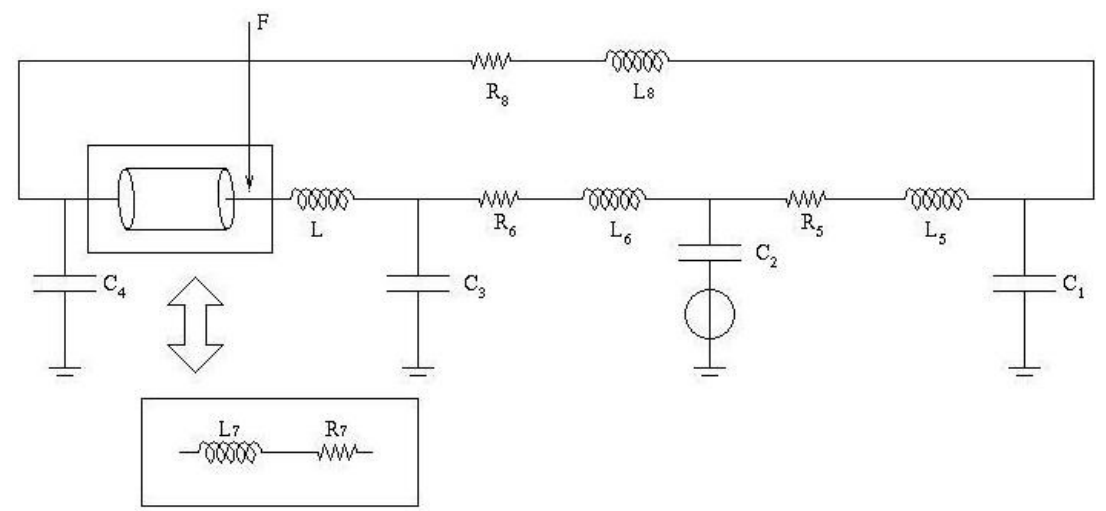

Figure 6: Network.

\section{AcKnowledgments}

The authors wish to thank L. Formaggia, A. Veneziani, J.F. Gerbeau and C. Prud'homme for their relevant suggestions.

The second author has been supported by European Union under contract HPRN $-C T-2002-002670$.

The numerical solutions obtained were developed in $C++$ in the scope of the finite element library $L i f e V$.

\section{REFERENCES}

1] M. Fernández, V. Milisisic and A. Quarteroni, Analysis of a geometrical multiscale blood flow model based on the coupling of ODE's and hyperbolic PDE's, SIAM J. on Multiscale Model. Simul., (2004). Accepted.

[2] L. Formaggia, J.F. Gerbeau, F. Nobile and A. Quarteroni, Numerical treatment of defective boundary conditions for the Navier-Stokes equations, SIAM Journ. Num. Anal., 40, 376-401 (2002).

[3] A. Gauthier, F. Saleri and A. Veneziani, A fast preconditioner for the incompressible Navier-Stokes equations, Computing and Visualization in Science, 6, 105-112 (2004).

[4] L. Formaggia and A. Veneziani, Reduced and multiscale models for the human cardiovascular system, Lecture notes VKI Lecture Series 2003-07, Brussels 2003.

[5] L. Formaggia, J.F. Gerbeau, F. Nobile and A. Quarteroni, On the coupling of 3D and 1D Navier-Stokes equations for flow problems in compliant vessels, Comput. Methods Appl. Mech. Engrg., 191, 561-582 (2001)

[6] L. Formaggia, D. Lamponi and A. Quarteroni One dimensional models for blood flow in arteries, Juornal of Engineering Mathematics, 47, 251-276 (2003)

[7] L. Formaggia, D. Lamponi, M. Tuveri and A. Veneziani, Numerical modeling of 1D arterial networks coupled with a lumped parameters description of the heart, Internal technical report $n^{\circ} 31$, Politecnico di Milano (2004).

[8] L. Formaggia, F. Nobile, A. Quarteroni and A. Veneziani, Multiscale modeling of the circulatory system: a preliminary analysis, Computing and Visualizations in Science, 2, 75-83 (1999)

[9] J. Heywood, R. Rannacher and S. Turek, Artificial boundaries and flux and pressure conditions for the incompressible NavierStokes equations, Int. Journ. Num. Meth. Fluids 22, 325-352 (1996).

[10] V. Milisiić and A. Quarteroni, Analysis of lumped parameter models for blood flow simulations and their relation with 1D models, ESAIM: Mathematical Modelling and Numerical Analysis,38, 613-632, (2004).

[11] A. Quarteroni and L. Formaggia, Handbook of Numerical Analysis, volume XII, chapter Mathematical modelling and numerical simulation of the cardiovascular system, Elsevier, Amsterdam, (2002).

[12] A. Quarteroni, M. Tuveri and A. Veneziani, Computational vascular fluid dynamics: problems, models and methods, Computing and Visualizations in Science 2, 163-197 (2000)

[13] A. Quarteroni, S. Ragni and A. Veneziani, Coupling between lumped and distributed models for blood flow problems, Computing and Visualizations in Science 4, 111-124 (2001)

[14] A. Quarteroni and A. Veneziani, Analysis of a geometrical multiscale model based on the coupling of ODE's and PDE's for blood flow simulation, SIAM j. Multiscale Model. Sim., 1, 173-195, (2003).

[15] J. Ottesen, M. Olufsen, J. Larsen, Applied Mathematical Models in Human Physiology, SIAM Monographs on Mathematical Modeling and Computation, (2003). 
[16] A. Veneziani and C. Vergara, Flow rate defective boundary conditions in haemodinamics simulations, Int. Journ. Num. Meth. Fluids (2004). Accepted.

[17] A. Quarteroni, F. Saleri and A. Veneziani, Factorization methods for the numerical approximation of Navier-Stokes equations, Comp. Methods Appl. Mech. Engrg. 188, 505-526 (2000).

[18] A. Veneziani, Block factorized preconditioners for high-order accurate in time approximation of the Navier-Stokes equations, Num. Meth. PDE's 19, 487-510 (2003) 